\title{
Erratum: Immune responses to commensal and environmental microbes
}

Eric G Pamer

Nat. Immunol. 8, 1173-1178 (2007); published online 19 October 2007; corrected after print 19 October 2007

In the version of this article initially published, the citations for Figure 1 and Figure 2 are incorrect. The citations for Figure 2a, 2b and $2 \mathrm{c}$ should be $1 \mathrm{a}, 1 \mathrm{~b}$ and $1 \mathrm{c}$; the citations for Figure 1 should be Figure 2. The error has been corrected in the HTML and PDF versions of the article.

\footnotetext{
Corrigendum: Comprehensive epigenetic profiling identifies multiple distal
을
regulatory elements directing transcription of the gene encoding interferon- $\gamma$

Jamie R Schoenborn, Michael O Dorschner, Masayuki Sekimata, Deanna M Santer, Maria Shnyreva, David R Fitzpatrick, John A Stamatoyannopoulos \& Christopher B Wilson

Nat. Immunol. 8, 732-742 (2007); published online 3 June 2007; corrected after print 16 November 2007

In the version of this article initially published, labels in Figures 2 and 4 are incorrect (as is the relevant text for Figure 4 ), and a reagent is incorrectly identified in Methods. In Figure 2, the label "IfngCNS+54" should be "IfngCNS+55." For Figure 4, the primers used to analyze the intronic region of Ifng for CpG methylation after bisulfite treatment amplify intron 1; thus, the label above the sixth column in Figure 4a should be "Ifng intron 1" and the accompanying text on page 736, column 1, should read "intron 1" on lines 3 and 13. In the Methods section, page 740 column 2, the end of line 23 should read "rabbit immunoglobulin G." The errors have been corrected in the PDF version of the article.
} 